\title{
Star-Formation Histories of Resolved Galaxies in the Local Volume
}

\author{
Alessandra Aloisi \\ Space Telescope Science Institute, \\ 3700 San Martin Drive, Baltimore, MD-21218, USA \\ email: aloisi@stsci.edu
}

\begin{abstract}
Color-magnitude diagrams of resolved stellar populations are the most powerful tool to derive the star formation histories of galaxies. In this review the method to infer star formation histories by comparing synthetic color-magnitude diagrams to deep, high-precision, multi-color photometry is briefly outlined, and our current knowledge of the star formation history of systems within the local Volume is summarized.
\end{abstract}

Keywords. Local Group, Magellanic Clouds, galaxies: stellar content, galaxies: distances and redshifts, galaxies: evolution

\section{Introduction}

With the term of resolved galaxies we will refer to galaxies near enough to be resolved into single stars with high-angular resolution (space) telescopes, like the Hubble Space Telescope (HST) which has been a game changer in this field over the past two decades.

Thanks to HST, stellar populations have been resolved in several nearby galaxies, including dwarf and massive galaxies, early- and late-type galaxies, in the Local Group and beyond. Only the filters F555W/F606W (equivalent to Johnson/broadband $V$ ) and F814W (equivalent to Johnson-Cousins $I$ ) of the HST imaging cameras (e.g., WFC2, ACS/WFC, and WFC3 for 0.05"-0.1" per pixel) have been really exploited so far for this purpose, thanks to their higher sensitivity to both young and old stars.

\section{Reconstructing the Star-Formation Histories of Galaxies}

A galaxy star-formation history $(\mathrm{SFH})$ is a detailed description of how, when, and where the galaxy formed its stars. SFHs of galaxies can be inferred from the colormagnitude diagrams (CMDs) of their resoved stars by applying the synthetic CMD method. This method consists in simulating a CMD by determining the variation of the star-formation rate (SFR) within the look-back time reached by the available photometry. Theoretical CMDs are created via Monte-Carlo based extractions of single stars from stellar evolution tracks, or isochrones, for a variety of star formation (SF) laws, IMFs, binary fractions, age-metallicity relations, distance, extinction, etc. Observational factors, including photometric errors, incompleteness, and stellar crowding, are also estimated and included in the procedure to fully reproduce an observed CMD. A combination of assumed parameters is acceptable only if the resulting synthetic CMD satisfactorily reproduces all the main features of the observational diagram (Fig. 1, left).

With sufficient investment of HST time, individual stars can be resolved down to the oldest main sequence turn-off (MSTO) in all galaxies of the Local Group and the resulting CMD can be used to infer their SFH over the entire Hubble time. If the oldest MSTO is not reached, then the look-back time depends on which CMD features can be resolved. 

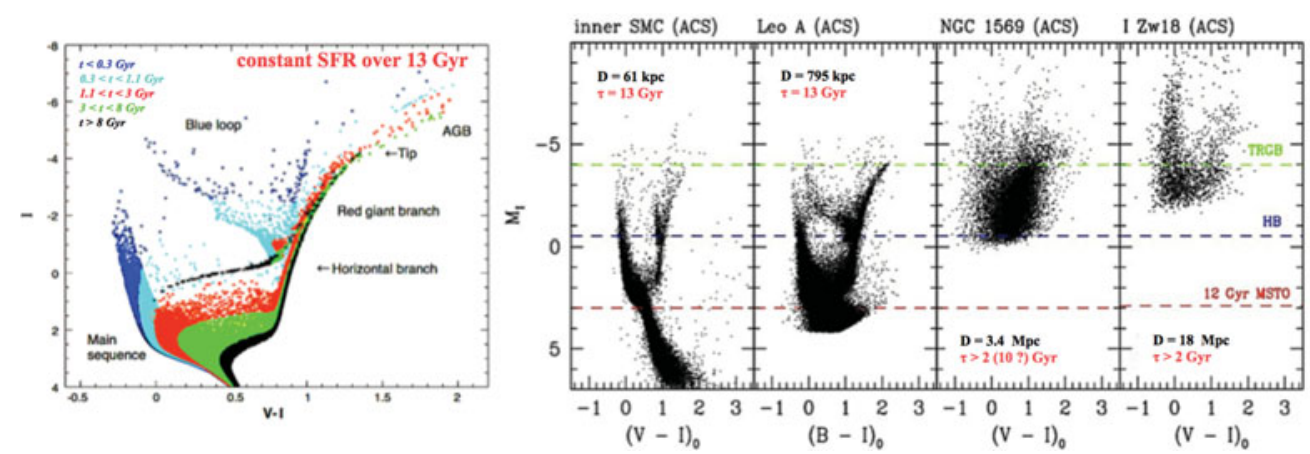

Figure 1. Left: Theoretical $I$ vs. $V-I$ CMD for a galaxy that has formed stars constantly over the past 13 Gyr (Tolstoy 2011). Key stellar evolution phases indicating the presence of stellar populations of different ages, are also marked. Right: Effect of distance on depth of photometry in the observed CMDs of dwarf galaxies imaged with HST/ACS. The position of key CMD stellar features (indicated as horizontal dashed lines) can be used as indicator of the reachable look-back times and stellar ages within each galaxy (Courtesy: F. Annibali).

The Main Sequence (MS) contains stars with different ages that are nicely spread out in color, and older ages can be reached depending on the depth of the photometry. The Horizontal Branch (HB), an almost horizontal sequence at $I \sim 0$, only contains (metal-poor) stars $>10$ Gyr old and it is hard to interpret in terms of SFH, except for an indication that ancient stellar populations are present. The Red Giant Branch (RGB) contains stars $>1-2$ Gyr old, but without further information it is impossible to be quantitative about the SFH because of the age-metallicity degeneracy. The Asymptotic Giant Branch (AGB) above the tip of the Red Giant Branch (TRGB) is a mix of intermediate-age stars. Very young stars in the upper MS and Blue Loops (BLs) are much brighter, and obtaining the SFH over the last $1 \mathrm{Gyr}$, especially in actively star-forming galaxies, is possible well beyond the Local Group (Fig. 1, right).

\section{Star-Formation Histories in the Local Group}

The Local Group (LG) contains $\sim 50$ galaxies within a radius of $\sim 1 \mathrm{Mpc}$, i.e. the distance from us of the most distant galaxies that are still dynamically part of the same group of galaxies of the Milky Way (MW). The LG mass is only dominated by 4 galaxies: the 3 spiral galaxies MW, Andromeda (M31), and Triangulum (M33), and the irregular Large Magellanic Cloud (LMC). All other galaxies, including the Small Magellanic Cloud (SMC), are less massive dwarfs (dEs, dSphs, dSph/dIrrs, and dIrrs), where a dwarf is defined as a galaxy with $M_{B}>-18$ mag. Resolved stellar population studies of the MW are limited to small nearby regions and star clusters due to our location. All other LG galaxies have been extensively studied and resolved into single stars thanks to their vicinity.

\subsection{Large and Small Magellanic Clouds}

The first global analysis of the resolved stars and SFHs of the LMC $\left(8.5^{\circ} \times 7.5^{\circ}\right)$ and SMC $\left(4^{\circ} \times 4.6^{\circ}\right)$ over their entire history is from the $U B V I$ Magellanic Cloud Photometric Survey (MCPS) completed with the 1-m Swope Telescope (0.7" per pixel) at the Las Campanas in Chile (Fig. 2).

Following an initial burst of SF, both the LMC and SMC experienced a quiescent epoch from $\sim 10$ to 3-5 Gyr ago. SF activity then resumed in both these MW satellites and has proceeded until the current time. The peaks in the SFH of the LMC at $0.5 \mathrm{Gyr}$ and 2-3 Gyr are nearly coincident with similar peaks in the SFH of the SMC, suggesting 

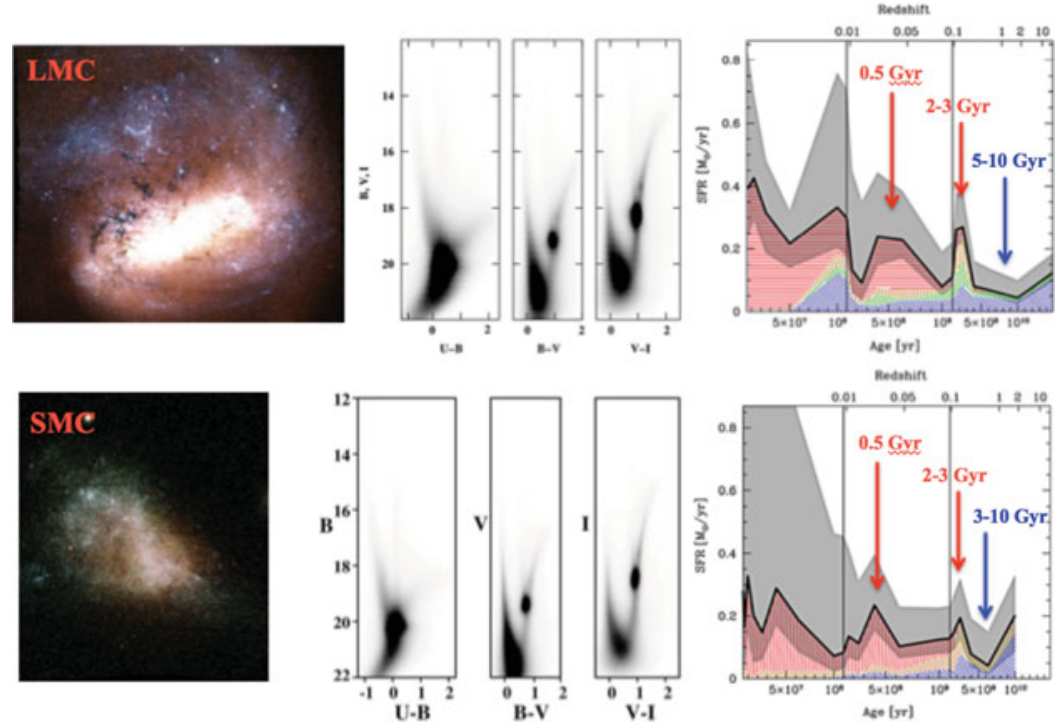

Figure 2. Top Panels: Multi-color MCPS image (left), CMDs in the UBVI filters (middle), and global SFH (right) of the LMC. Bottom Panels: Same but for the SMC (Harris \& Zaritsky 2004, 2009). SFR vs. age is shown as thick black line (right panels); uncertainty on the fit is shown as gray envelope. The metallicity distribution at each age is shown by the mix of colors $\left(Z=0.001,0.0025,0.004\right.$, and $0.008 \mathrm{Z}_{\odot}$ in blue, green, orange, and red, respectively).
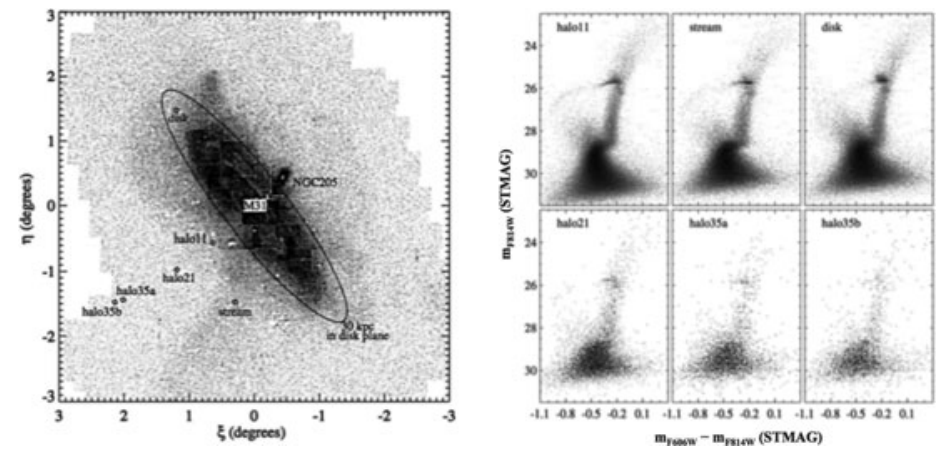

Figure 3. Left: M31 stellar density map with the 6 HST fields labelled. An ellipse marks the area within $30 \mathrm{kpc}$ of the galactic center in the inclined disk plane. Right: $I$ vs. $V-I$ CMDs of the 6 fields from Brown et al. (2009).

a joint history for these galaxies extending back at least several Gyr. These peaks are coincident with past peri-galactic passages with the MW. About $50 \%$ of the LMC and SMC mass was assembled 5-8 Gyr ago. The SFH of the field stars in the LMC and SMC is consistent with their cluster formation history (Harris \& Zaritsky 2004, 2009).

\subsection{The Andromeda Galaxy (M31)}

Several stellar fields were observed with HST in the halo, disk, and stream of M31 over the past decade (e.g., Brown et al. 2009 and references therein; Fig. 3). The $11 \mathrm{kpc}$ halo, the stream, and the disk share an extended SFH. The halo has $\sim 60 \%$ of the mass in old (11-13 Gyr) metal-poor $([\mathrm{Fe} / \mathrm{H}]<-1 \mathrm{dex})$ stars and $\sim 40 \%$ in intermediate-age $(6-8 \mathrm{Gyr})$ metal-rich $([\mathrm{Fe} / \mathrm{H}]>-0.5$ dex $)$ stars likely connected to the extended outer disk (rather than the outer halo). The $11 \mathrm{kpc}$ halo and the stream also have remarkably similar CMDs, 

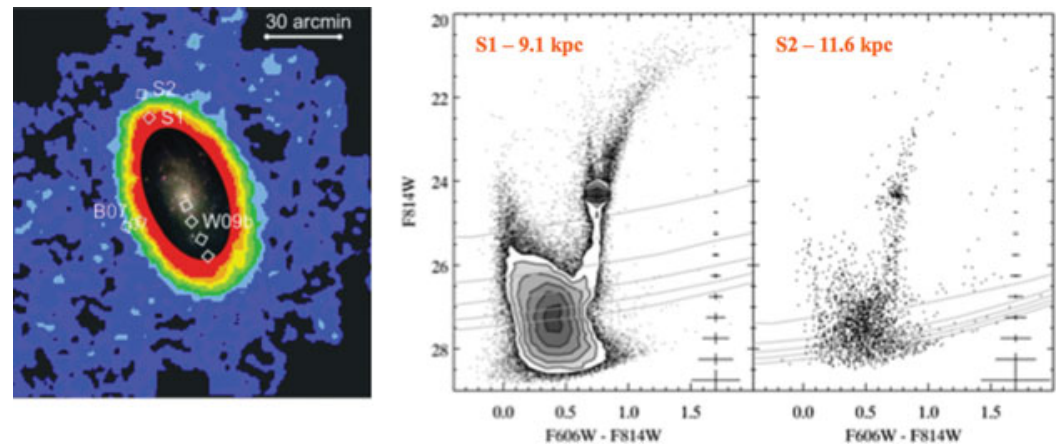

Figure 4. Left: Contour map of M33 RGB stellar surface density from the Isaac Newton Telescope Wide Field Camera (INT/WFC) survey with an optical image of the inner disc overlaid. All HST fields observed are labelled, with the two halo fields at 9.1 and $11.6 \mathrm{kpc}$ indicated as S1 and S2. Right: $I$ vs. $V-I \mathrm{CMD}$ of $\mathrm{S} 1$ and $\mathrm{S} 2$. Median errors are on the right. Solid gray lines from top to bottom denote $90 \%, 80 \%, 70 \%, 60 \%$, and $50 \%$ completeness (Barker et al. 2011).

even if the stream is $\sim 1$ Gyr younger on average. This suggests pollution of the halo by the stream progenitor that has been likely accreted by M31. The disk is instead younger (4-8 Gyr) and more metal-rich $([\mathrm{Fe} / \mathrm{H}] \sim-0.2 \mathrm{dex})$. The 21 and $35 \mathrm{kpc}$ halo fields are mostly old, with an age spread around the mean value of $\sim 10$ and $11 \mathrm{Gyr}$, respectively, and a wide range in metallicity with a mean $[\mathrm{Fe} / \mathrm{H}]$ value of $\sim-0.7$ and -1 dex, respectively. The extended halo of M31 thus cleary shows evidence of hierarchical assembly.

\subsection{The Triangulum Galaxy (M33)}

While several HST observations exist of M33, only a couple of fields (S1 and S2) at 9.1 and $11.6 \mathrm{kpc}$ in the halo of the galaxy along its northern major axis, have been analyzed with the method of the synthetic CMDs to infer their SFH (Barker et al. 2011; Fig. 4). The $9.1 \mathrm{kpc}$ halo field resulted on average younger ( $\sim 3 \mathrm{Gyr}$ old $)$ and more metal-rich $([\mathrm{M} / \mathrm{H}] \sim-0.5 \mathrm{dex})$ than the $11.6 \mathrm{kpc}$ field which is older $(\sim 7$ Gyr old $)$ and more metal-poor $([\mathrm{M} / \mathrm{H}] \sim-0.8 \mathrm{dex})$. The $\mathrm{SFH}$ of the $9.1 \mathrm{kpc}$ halo field reveals how M33 inner disk has undergone an inside-out growth.

\subsection{Dwarf Galaxies in the Local Group}

All LG dwarf galaxies have been extensively observed with HST and show different CMDs, with dSphs lacking gas and recent SF, dSph/dIrr containing some gas but not recent SF, and dIrr having gas and undergoing SF. While each galaxy has its unique SFH extending over many Gyr, they all began forming stars $\sim 13$ Gyr ago. The majority shows no sign of pause due to reionization $\sim 12.6 \mathrm{Gyr}$ ago $(\mathrm{z} \sim 6)$. The SFHs of LG dwarfs can be used to validate galaxy formation and evolution models (Weisz et al. 2014; Fig. 5).

\section{Star-Formation Histories beyond the Local Group}

Going beyond the LG means going to distances $>1 \mathrm{Mpc}$ where resolution of single stars becomes more challenging. While spirals are included in the LG, going beyond means to find other types of galaxies. The closest giant elliptical is Centaurus A (NGC 5128) in the Centaurus group at $D=3.8 \mathrm{Mpc}$. Active galaxies including galaxies undergoing strong SF like, e.g., starbursts and blue compact dwarf galaxies (BCDs), are also only found beyond the LG. The closest starburst is NGC 1569 at $D=3.4 \mathrm{Mpc}$ in the IC 342 group, and the closest most metal-poor BCD is DDO 68 in a void at $D=12.1 \mathrm{Mpc}$ (Fig. 6). 

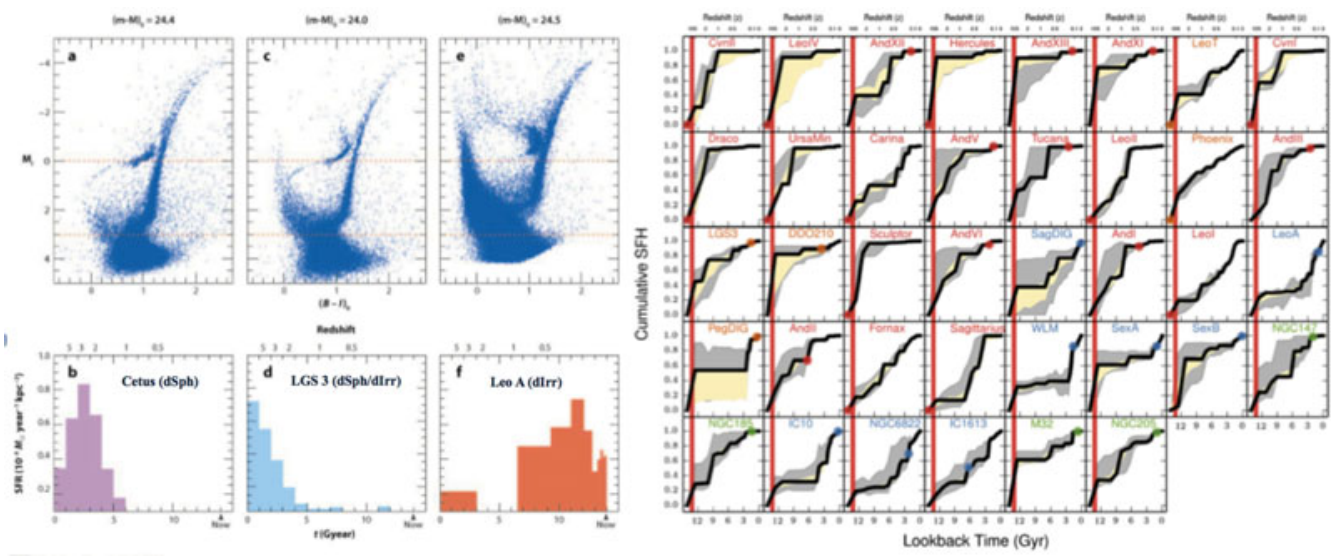

Figure 5. Left: HST/ACS $I$ vs. $B-I$ CMDs and SFHs for 3 LG dwarf galaxies from the LCID project (Tolstoy, Hill, \& Tosi 2009). Right: HST/WFPC2 cumulative SFHs in 38 LG dwarfs sorted by increasing luminosity (Weisz et al. 2014). Solid black lines are best-fit SFHs, yellow and gray envelopes represent $1 \sigma$ uncertainties from random and systematic errors. Colored dots are approximate MSTO age of the CMD used to measure the SFH. Dwarfs are color-coded by morphological type $(\mathrm{dSphs}=\mathrm{red}$; dIrrs = blue; dTrans = orange; dEs = green $)$. Red bands indicate the epoch of re-ionization. The majority of LG dwarfs did not form the bulk of their stellar mass prior to reionization, except for And V, And VI, And XIII, Hercules, and Leo IV.

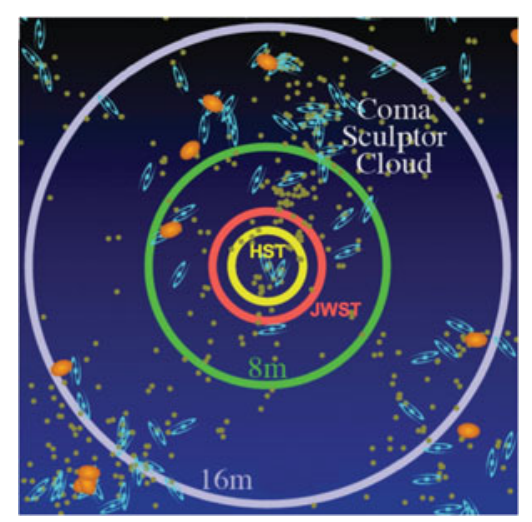

Figure 6. Map of nearby galaxies color-coded by morphological type (Blue = large spiral, Orange $=$ large elliptical, Olive $=$ dwarf galaxy). Circles show how far away a $\mathrm{L}_{\odot}$ star can be detected at $\mathrm{S} / \mathrm{N}=5$ in two bands in a total exposure of 100 hours (Yellow $=2.4-\mathrm{m}$ (HST), Red $=6.5-\mathrm{m}$ (JWST), Green $=8-\mathrm{m}$, Purple $=16-\mathrm{m}$ space telescopes). Spirals, ellipticals, and dwarfs come into view beyond $1 \mathrm{Mpc}$, corresponding to the yellow circle (Postman et al. 2010).

\subsection{The closest Giant Elliptical Galaxy: Centaurus A (NGC 5128)}

At $D=3.8 \mathrm{Mpc}$, NGC 5128 can only be resolved into single stars with HST. Several halo fields were observed with WFPC2 down to the RGBT. The deepest field located at $\sim 37 \mathrm{kpc}$ from the galaxy center, was instead observed with ACS/WFC and its CMD revealed the Red Clump (RC) and $\mathrm{HB}$ in a giant E/SO galaxy for the first time (Rejkuba et al. 2005; Fig. 7). Combining the metallicity-sensitive color of the RGB with the ageand metallicity-sensitive features of the AGB and RC bumps, an average age of $\sim 8$ Gyr was inferred. An average metallicity of $[M / H] \sim 0.6$ dex indicates that the halo is metal-rich all the way out. No SFH was inferred from the observed CMDs of this galaxy. 

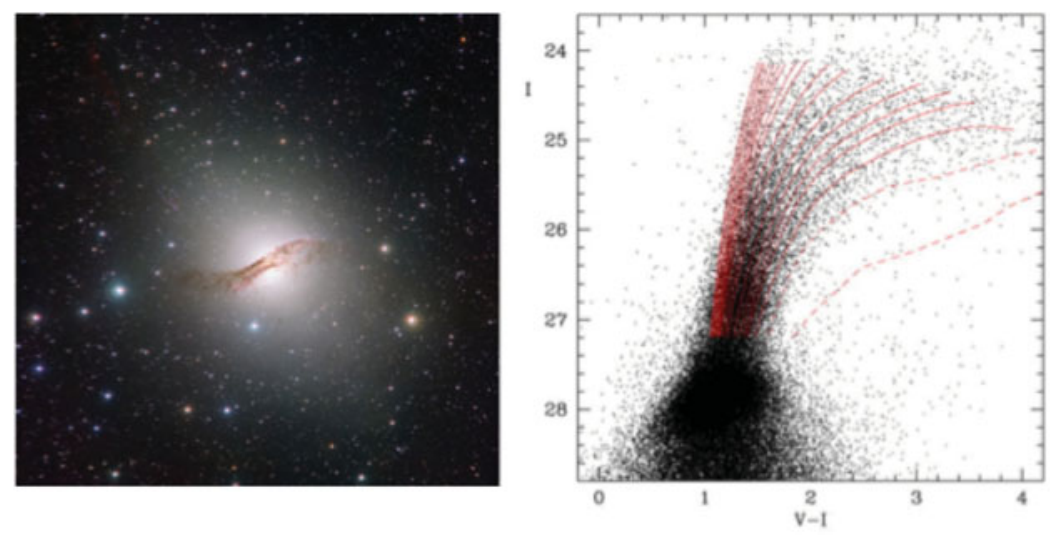

Figure 7. Left: Multi-color image of NGC 5128 obtained with the Wide Field Imager attached to the MPG/ESO 2.2-m telescope at the La Silla Observatory in Chile (Credit: ESO). Right: $I$ vs. $V-I$ CMD of the deepest halo field in NGC 5128 located at $37 \mathrm{kpc}$ (outside of image bottom left). The CMD displays a wide RGB, an AGB, and a RC; solid and dashed lines are various theoretical models at different metallicities (Rejkuba et al. 2005).
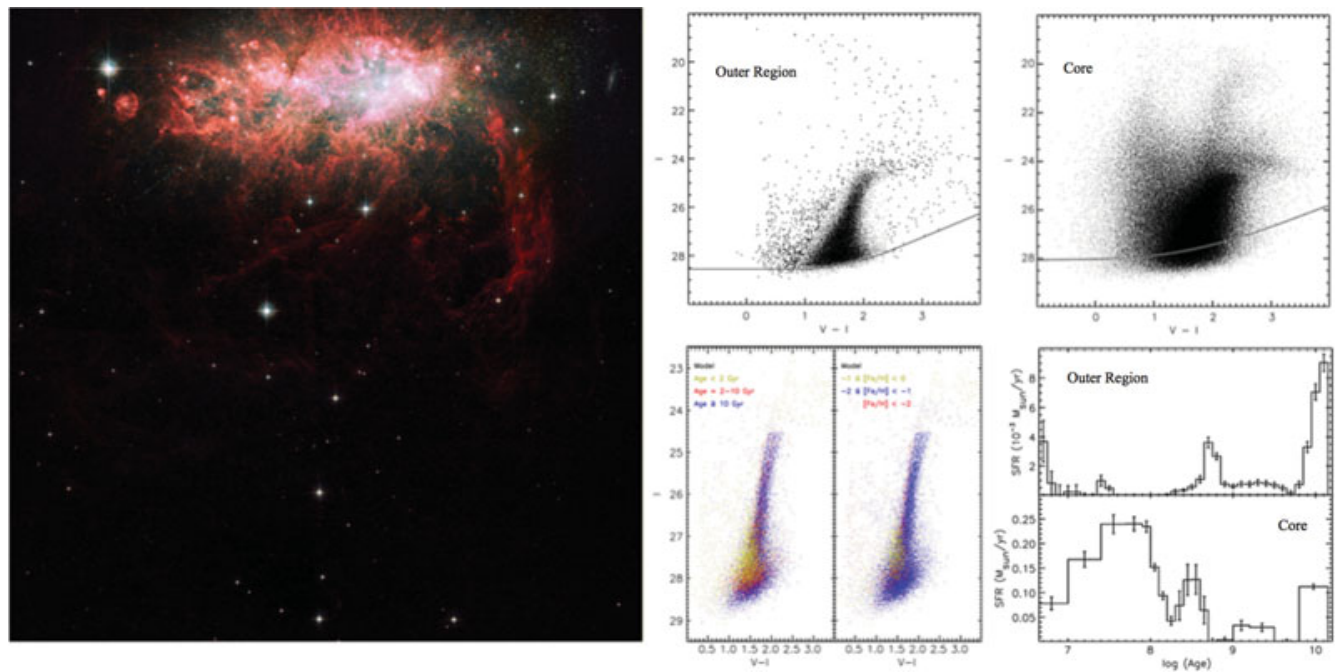

Figure 8. Left: ACS/WFC multi-color image of NGC 1569. Top Middle and Right: $I$ vs. $V-I$ CMD for the outer region (halo) and the core. $20 \%$ completeness is shown as solid line. Features from young, massive, MS and evolved stars dominate the core. Only old RGB stars are visible in the outer region. Bottom Middle: Synthetic CMD for the outer region of NGC 1569. Different colors indicate separate ages (left) and metallicities (right). Bottom Right: SFR vs. age for the outer (Grocholski et al. 2012) and core-dominated (McQuinn et al. 2010) regions.

\subsection{The closest (Dwarf Irregular) Starburst Galaxy: NGC 1569}

At $D=3.4 \mathrm{Mpc}$, NGC 1569 is a dwarf irregular with a metallicity of $\sim 0.25 \mathrm{Z}_{\odot}$ currently undergoing a strong starburst. Its halo was resolved into single stars with ACS/WFC down to the RC/HB by our group (Grocholski et al. 2012; Fig. 8). This CDM stellar feature was used to disentangle the age-metallicity degeneracy of the RGB in the simulations. The observed RGB width required a spread in metallicity (in addition to a spread in ages) similar to that of the MW halo. The oldest strongest SF episode at $10 \mathrm{Gyr}$ accounts for most of the mass. A relatively low level of SF persisted between 10 and 1 Gyr, until the onset of a SF burst at $\sim 0.3-0.7$ Gyr. The new distance and radial velocity 

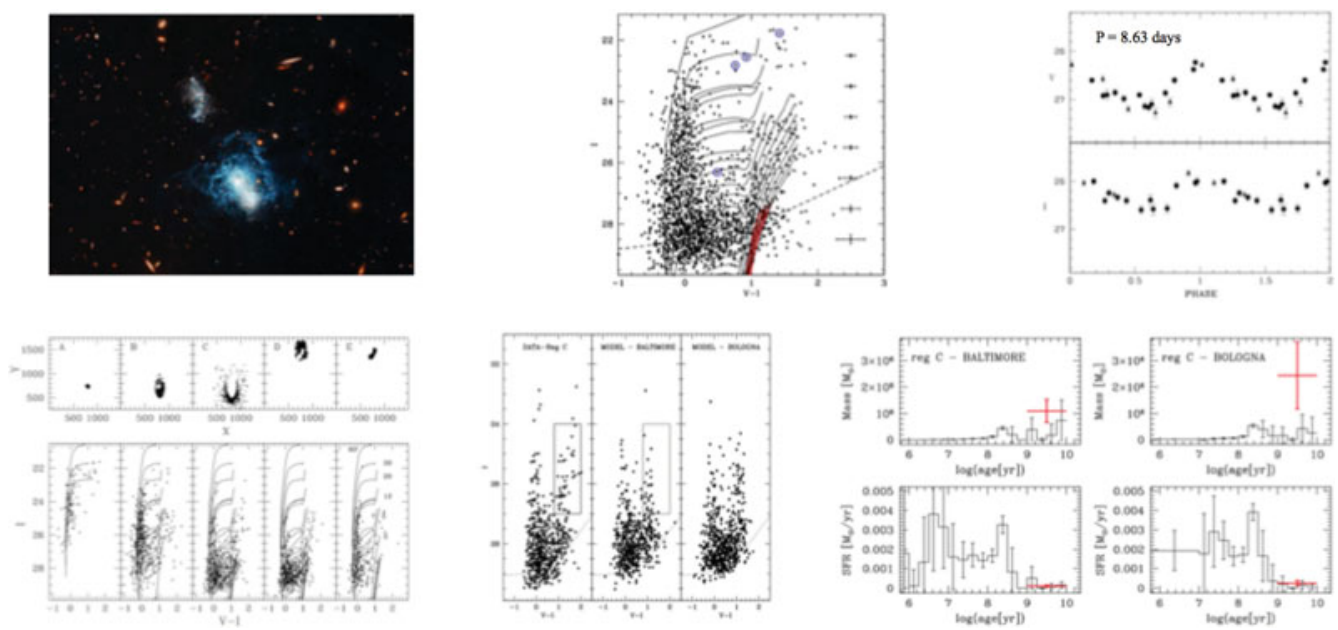

Figure 9. Top Left: ACS/WFC multi-color image of I Zw 18. Top Middle: $I$ vs. $V-I$ CMD with stellar evolution tracks (RGB tracks in red). Median errors are on the right. Dashed curve is $50 \%$ completeness. TRGB gives $D=18 \pm 2 \mathrm{Mpc}$. Four Cepheids (blue circles) give $D=19 \pm 2 \mathrm{Mpc}$. Top Right: $V$ and $I$ light curves (corrected for foreground extinction) for the faintest 8.63 day Cepheid. Bottom Left: Spatial distribution of stars in regions A-E and corresponding CMDs with stellar evolution tracks for $60,30,20,12,7,5,2,1,0.8$, and $0.6 \mathrm{M}_{\odot}$. Bottom Middle: Observed and synthetic CMDs for region $\mathrm{C}$ obtained with two different codes (Baltimore and Bologna). Dotted curve is $20 \%$ completeness. Box indicates TP-AGB stars. Bottom Right: Stellar mass and SFR vs. age for region $\mathrm{C}$ with Baltimore and Bologna codes. Thick red line at oldest ages indicates the values obtained using only one 1-10 Gyr bin.

suggest that NGC 1569 moved through the IC 342 group over the past few Gyrs The interaction with the group galaxy UGCA 92 may be driving the most recent burst $(<10$ Myr old).

\subsection{The Famous Most Metal-Poor BCD at the Edge of the Local Volume: I Zw 18}

I Zw 18 is the farthest galaxy ever been resolved into single stars with HST in the Local Volume $(\sim 20 \mathrm{Mpc})$. With the lowest metal abundance ever observed $\left(\sim 1 / 50 \mathrm{Z}_{\odot}\right)$, it is considered the closest analog to primordial galaxies. ACS/WFC time-series observations were obtained by our group, and faint RGB stars that had been previously elusive, were resolved for the first time. A new distance was inferred via TRGB and Cepheids, indicating that the galaxy is much farther away at $\sim 18 \mathrm{Mpc}$ (Aloisi et al. 2007; Fig. 9). Simulations confirm that I Zw 18 started forming stars $~ 13$ Gyr ago (Annibali et al. 2013).

\subsection{The Closest Newly Discovered Most Metal-Poor BCD : DDO 68}

DDO 68 is a BCD recently classified as metal-poor as I Zw 18, but a factor of 2-3 closer. Deep ACS/WFC observations by our group down to the RGB give a new distance of $D=12.1 \mathrm{Mpc}$, making the galaxy a few Mpc farther away than previous estimates. The CMDs of regions 1-4 show a gradient in stellar populations, with young stars mostly in the center, and old ( $>1$ Gyr) RGB stars everywhere. A substantial population of $<10$ Myr old stars is in regions 2 and 3 where most of the H II regions are also located.

Particularly interesting is region 3 due to the presence of $\mathrm{H}$ II regions on a stream of stars suggesting accretion. Synthetic CMDs indicate a very high costant SF activity bewteen $\sim 10$ Myr and 1 Gyr. A more recent, stronger, and still active burst is also detected at $\sim 1$ Myr. $55 \%$ of the total mass was produced $>1$ Gyr ago (Sacchi et al. 2016, in 

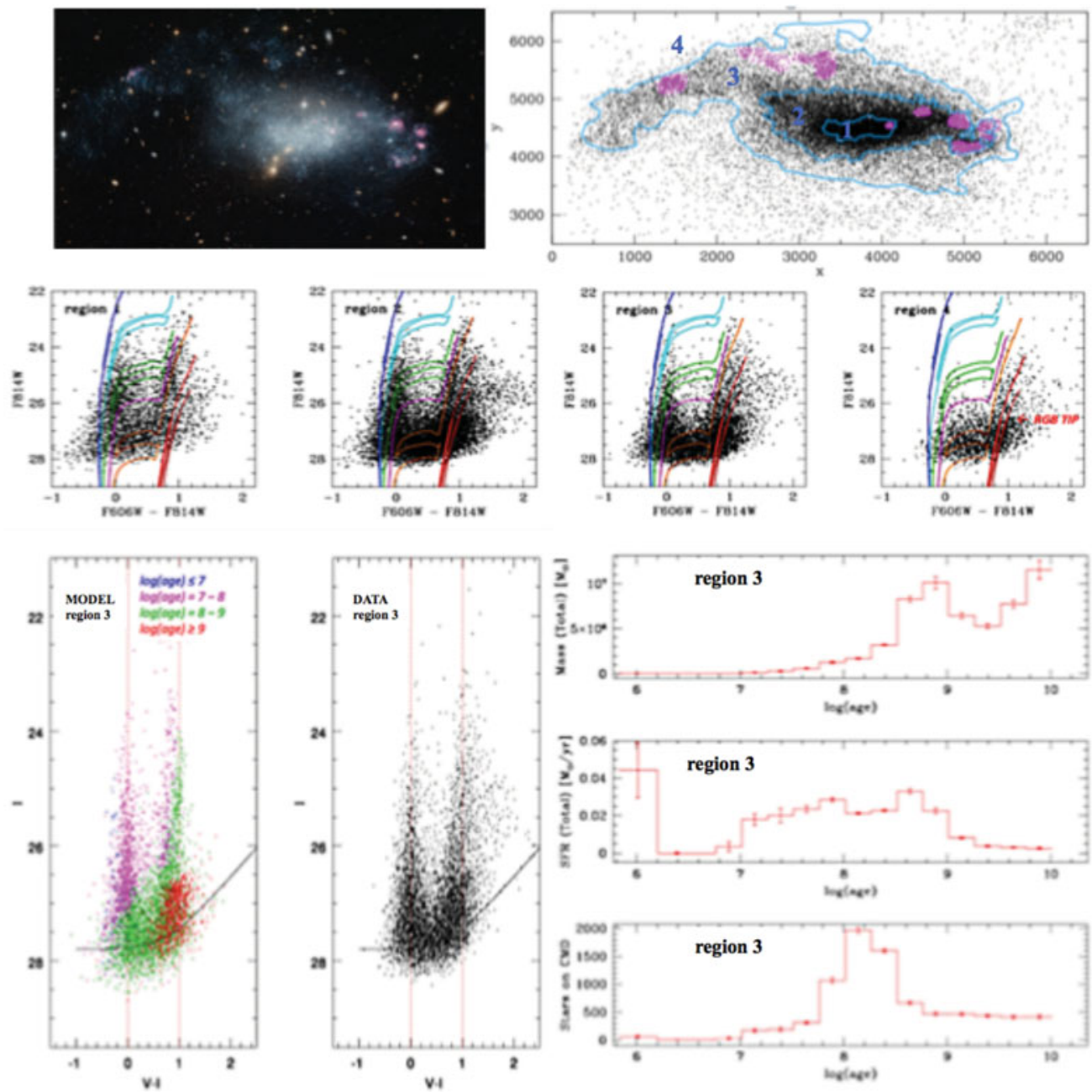

Figure 10. Top Left: ACS/WFC multi-color image of DDO 68. Top Right: Map of stars photometrized in $V$ and $I$. Cyan contours define regions 1-4. Magenta contours indicate $\mathrm{H}$ II regions from $H \alpha$ imaging. Middle: $I$ vs. $V-I$ CMDs of the four regions with isochrones overlaid for 10, 20, 50, 100, $200 \mathrm{Myr}, 2$ and 13 Gyr (top to bottom). Bottom Left: Observed and synthetic CMD of region 3. Bottom Right: Stellar mass, SFR, and number of stars vs. age for region 3.

preparation; Fig. 10). A low-mass H I companion of DDO 68 in the direction of the stream of stars of region 3 , has been very recently located at $\sim 42 \mathrm{kpc}$ (Cannon et al. 2014).

\section{Summary and Conclusions}

The SFH of resolved galaxies is a powerful tool to understand their formation and evolution, including interactions with the surrounding environment. All galaxies resolved into single stars with HST within the Local Volume have revealed stars as old as the lookback times probed by the data. The SFHs of LG dwarf galaxies with similar morphology vary widely, suggesting that many factors are at play in shaping the evolution of these objects. Studies of the halo in the closest spirals and ellipticals support the hierarchical galaxy formation scenario, while the SFH of active dwarf galaxies outside the LG suggest that interactions play a key role in enhancing their present SF activity. 


\section{References}

Aloisi, A., Clementini, G., Tosi, M., Annibali, F., Contreras, R., Fiorentino, G., et al. 2007, ApJ (Letters), 667, 151

Annibali, F., Cignoni, M., Tosi, M., van der Marel, R. P., Aloisi, A., \& Fiorentino, G. 2013, AJ, 150,143

Barker, M. K., Ferguson, A. M. N., Cole, A. A., Ibata, R., Irwin, M., Lewis, G. F., et al. 2011, MNRAS, 410, 504

Brown, T. M., Smith, E., Ferguson, H. C., Guhathakurta, P., Kalirai, J. S., Kimble, R. A., et al. 2009, ApJ (Supplement), 184, 152

Cannon, J. M., Johnson, M., McQuinn, K. B. W., Alfvin, E. D., Bailin, J., Ford, H. A., et al. 2014, ApJ, 787, 1

Grocholski, A. J., van der Marel, R. P., Aloisi, A., Annibali, F., Greggio, L., \& Tosi, M. 2012, $A J, 143,117$

Harris, J. \& Zaritsky, D. 2004, $A J, 127,1531$

Harris, J. \& Zaritsky, D. 2009, AJ, 138, 1243

McQuinn, K. B. W., Skillman, E. D., Cannon, J. M., Dalcanton, J., Dolphin, A., HidalgoRodríguez, S., et al. 2010, ApJ, 721, 297

Postman, M., Brown, T., Sembach, K., Giavalisco, M., Traub, W., Stapelfeldt, K., Calzetti, D., Oegerle, W., et al. 2010, Proc. of SPIE, 7731, 77312K-1

Rejkuba, M., Greggio, L., Harris, W. E., Harris, G. L. H., \& Peng, E. W. 2005, ApJ, 631, 262

Tolstoy, E. 2011, Science, 333, 176

Tolstoy, E., Hill, V., \& Tosi, M. 2009, ARAA, 47, 371

Weisz, D. R., Dolphin, A. E., Skillman, E. D., Holtzman, J., Gilbert, K. M., Dalcanton, J. J., et al. 2014, ApJ, 789, 148 